\title{
Relative Expression of OCT4, SOX2 and NANOG in Oral Squamous Cell Carcinoma Versus Adjacent Non- Tumor Tissue
}

\author{
Fereshteh Baghai Naini ${ }^{1,2}$, Pouyan Amini Shakib ${ }^{1}$, Alireza Abdollahi ${ }^{3}$, Mahshid \\ Hodjat $^{2}$, Hadiseh Mohammadpour ${ }^{4}$, Neda Kardouni Khoozestani ${ }^{1 *}$
}

\begin{abstract}
Objective: Oral squamous cell carcinoma (OSCC) accounts for over $90 \%$ of oral neoplasms. Finding molecular markers for predicting prognosis is a high priority. The core transcription factors, OCT4, SOX2, and NANOG that regulate embryonic stem cell pluripotency have been implicated in progression of various malignancies. The predictive value of these markers and their role in the development of OSCC is still controversial. In this study, we therefore evaluated their expression in OSCCs and adjacent non-tumor tissue. Methods: A total of 60 frozen tumor and adjacent non-tumor tissue samples from 30 patients with OSCC were examined using quantitative reverse transcription polymerase chain reaction (qRT-PCR). Clinical and pathological data of patients including tumor stage, lymph node metastasis and tumor grade were also recorded. Results: Expression of SOX2 was significantly higher in adjacent non-tumor as compared to tumor tissue $(\mathrm{P}=0.04)$. No statistically significant differences were found for expression of OCT4 $(\mathrm{P}=0.50)$ and NANOG $(\mathrm{P}=0.68)$. Also, there was no significant association between expression of OCT4, SOX2, and NANOG and clinical or pathological data $(\mathrm{P}>0.05)$, although slightly higher values were noted in patients without lymph node metastasis. Conclusion: Based on the present data, decreased expression of SOX2 is correlated with carcinogenesis in the oral cavity and development of OSCC.
\end{abstract}

Keywords: OCT4- SOX2- NANOG- squamous cell carcinoma- gene expression

Asian Pac J Cancer Prev, 20 (6), 1649-1654

\section{Introduction}

It is known that $5 \%$ of all tumors develop in the head and neck region, out of which $50 \%$ occur in the oral cavity (Geum et al., 2013). The risk of recurrence of tumors in the oral cavity is relatively high compared to the tumors in other parts of the body (Liu et al., 2007). Presently, oral carcinomas are among the 10 most common malignancies in humans and has been reported as the major cause of morbidity, mortality and facial deformity worldwide (Bau et al., 2011; Bernier, 2011). Squamous cell carcinoma (SCC) accounts for over $90 \%$ of oral neoplasms (Sharma et al., 2010). The incidence of neck metastasis in oral cancer ranges from 19 to $34 \%$ and evidence shows that patients with neck metastasis have a poorer prognosis (Liu et al., 2007). Despite recent advances in cancer treatment, the rate of morbidity and mortality is still high in a considerable number of patients presenting with local recurrence, systemic metastasis or secondary tumors, which highlights the need for strategies for earlier detection and most effective treatment of cancer (Woolgar et al., 1995).

Tumors are comprised of subpopulations of cells, some of which display increased tumorigenic properties known as the cancer stem cell (CSC) (Al-Hajj and Clarke, 2004; Allegra et al., 2014; Islam et al., 2015; Reya et al., 2001). The presence of stem cell makers has been previously proven in different malignancies such as leukemia and breast cancer (Fillmore and Kuperwasser, 2008; Griffin and Lowenberg, 1986). The emergence of CSCs and detection of CSC markers in different tumoral tissues have profound impact on the development of molecular treatment modalities and replacing the conventional cancer treatments with more efficient alternative approaches (Al-Hajj and Clarke, 2004). So far, many key CSC markers have been introduced that are involved in the progression and development of OSCC (Al-Hajj and Clarke, 2004; Reya et al., 2001). The regulatory role of CD133 (Yu et al., 2016) and CD44 (Krishnamurthy and Nör, 2012) in self-renewal of CSC and tumor progression have been reported previously. Also studies focused on the core transcription factors OCT4, SOX2 and NANOG as the prognostic CSC markers in OSCC (Chiou et al., 2008; Vaiphei et al., 2014). A significant association has been reported between the expression of OCT4, SOX2, and NANOG with the grade of OSCC

${ }^{1}$ Department of Oral and Maxillofacial Pathology, Faculty of Dentistry, ${ }^{2}$ Dental Research Center, Dentistry Research Institute, ${ }^{3}$ Department of Pathology, Imam Khomeini Hospital Complex, ${ }^{4}$ Iran National Tumor Bank, Cancer Institute, Imam Khomeini Hospital Complex, Tehran University of Medical Science, Tehran, Iran.*For Correspondence: nkardouni@sina.tums.ac.ir 
(Chiou et al., 2008; Vaiphei et al., 2014).

OCT4, SOX2 and NANOG are the key transcription factors involved in pluripotency of embryonic stem cells and also regulating the fate of stem cells ( $\mathrm{Li}$ et al., 2015; Nichols et al., 1998; Ren et al., 2016). Their expression decrease during cells differentiation (Fu et al., 2016; Islam et al., 2015; Ren et al., 2016). They are also play critical role in cellular reprogramming of somatic cells.

Cancer initiation is the consequence of a multi-stage process of genetic changes, which occur prior to the histopathological occurrence of cancer. It is believed that these molecular changes can be detected even at initial stages when the tissue still appears histologically normal. Therefore, finding the key molecules undergoing changes at the very early stage of carcinogenesis can greatly enhance early detection of OSCC (Allegra et al., 2014; Islam et al., 2015).

This study sought to evaluate the expression of OCT4, SOX2 and NANOG CSC markers in OSCC and non-tumoral adjacent tissue specimens.

\section{Materials and Methods}

This observational study was conducted on biopsy samples taken from the tumoral tissue and adjacent non-tumoral tissue of 30 patients with primary diagnosis of OSCC retrieved from the tumor bank of the Pathology Department of Cancer Institute at Imam Khomeini Hospital. Patients were selected via non-probability convenience sampling. Demographic information of patients including age, sex, and ethnicity, the degree of differentiation of tumor and clinical stage of the tumor based on the recorded information were all collected in a checklist. The study was performed in accordance with the ethics board approval of Imam Khomeini Hospital.

\section{RNA extraction}

The mRNA was extracted from each specimen for real-time PCR to study the expression of OCT4, SOX2 and NANOG using RNeasy Mini kit (Qiagen, Germany) according to the manufacturer protocol. In brief, frozen tissue at $-80^{\circ} \mathrm{C}$ was cut by a sterile scalpel, diced on a plate and transferred to the homogenizing tube containing $1 \mathrm{~mL}$ RLT reagent. After shaking for 10 seconds, the lysate was centrifuged (1200rpm,) for $3 \mathrm{~min}$ at $4^{\circ} \mathrm{C}$ and the supernatant was transferred to a new tube containing $1 \mathrm{~mL}$ of $70 \%$ ethanol. Next, the mixture was passed through the RNeasy spin column followed by 2 washing step using $700 \mu \mathrm{L}$ RW1 and $500 \mu \mathrm{L}$ of RPE buffer. Finally, 30-50 $\mu \mathrm{L}$ RNase-free water was added to the spin column and after centrifugation, the isolated RNA was frozen at $-80^{\circ} \mathrm{C}$.

For assessing the RNA integrity, 1 to $2 \mu \mathrm{L}$ of the extracted RNA was subjected to $1.5 \%$ agarose gel electrophoresis with $142 \mathrm{~V}$ voltage for $15 \mathrm{~min}$ and the $28 \mathrm{~S}$ and $18 \mathrm{~S}$ ribosomal RNA bands were visualized by staining with EtdBr. Also, the purity of the extracted RNA was determined via the absorbance ratio of $260 \mathrm{~nm} / 280 \mathrm{~nm}$ using spectrophotometer (BioTek, USA).
cDNA synthesis

synthesis of cDNA was done using Scientific RevertAid First Strand cDNA Synthesis kit (Thermo, UK). Briefly, the extracted total RNA (5-10ng) were mixed with $1-2 \mu \mathrm{L}$ random hexamer primers and DEPC-treated water up to $10 \mu \mathrm{L}$. Next, the solution was heated at $65^{\circ} \mathrm{C}$ for $5 \mathrm{~min}$ and chilled at $4^{\circ} \mathrm{C} .10 \mu \mathrm{L}$ of the RT-PreMix (2x) was added to the solution and stored at $25^{\circ} \mathrm{C}$ for $10 \mathrm{~min}$ followed by incubation at $50^{\circ} \mathrm{C}$ for $60 \mathrm{~min}$. To finalize the reaction, the microtube was placed at $70^{\circ} \mathrm{C}$ for 10 min and cooled at $4^{\circ} \mathrm{C}$. Control primers and GAPDH RNA were used as the positive control of cDNA synthesis reaction.

\section{Real-time PCR (RT-PCR)}

To achieve a maximum level of sensitivity the annealing temperature was optimized prior to RT-PCR by thermal gradient PCR. The gradient PCR reaction was done for SOX2, OCT4 and Nanog in the total volume of $20 \mu 1$ Ampliqone (Taq DNA Polymerase Master Mix Red, Denmark) (Amplicon). In this process, $2 \mu \mathrm{L}$ (5-10pmols) template was used with $10 \mu \mathrm{l}$ amplicon, $1 \mu \mathrm{L}$ of each primer and sterilized DEPC water up to $20 \mu \mathrm{L}$. The PCR products were run on $2 \%$ agarose gel to analyze the products after visualization.

In the next step, RT-PCR was performed using SYBR green master mix (Takara Biotechnology, China). The primers used for performing PCR and RT-PCR were as follows: for Sox $2 \mathrm{~F}$ : 5'GACAGTTACGCGCACATGAA3' and R: 5'TAGGTCTG CGAGCTGGTCAT3'; for OCT4 F: 5'GGTATTCAGCCAAACGA CCA3' and R: 5'CACACTCGGACCACATCCTT3'; for NANOG F: 5'GTGATTTGTGGGCCTGA AGA3' and R: 5'ACACAGCTGGGTGGAAGAGA3'; for GAPDH F: 5'AATCCC ATCACCATCTTCCA3' and R: 5'TGGACTCCACGACATACTCA3'. Real-time PCR mixture contained $10 \mu 1$ of SYBR green master mix, $1 \mu 1$ of forward and reverse primers and $2 \mu$ of template DNA. The volume was adjusted to $20 \mu \mathrm{l}$ by DEPC water. The accuracy was ensured using the melting curve and gel electrophoresis (if required). GAPDH was used as the housekeeping gene in all reactions. RT-PCR was performed at least three times for each sample.

\section{Quantification of gene expression}

The cycle threshold (CT) method was used to quantify the level of gene expression. The relative difference was calculated using $2^{-\Delta \Delta \mathrm{CT}}$ formula where $\Delta \mathrm{CT}$ was the $\mathrm{CT}$ of the target gene (or calibrator), which was subtracted from the CT value of the house-keeping gene. In the current study, GAPDH was considered as the housekeeping gene and the calibrator was the adjacent non-tumoral tissue. The $\mathrm{CT}$ values of test samples (tumoral tissues) were compared with the CT values of the control (non-tumoral adjacent tissue) samples. To assess the statistical significance between tumoral and non-tumoral adjacent tissues the REST software was used.

\section{Statistical analysis}

Using descriptive statistics, the mean, standard deviation, median and interquartile range were reported for 
quantitative variables and frequency as well as percentage were reported for qualitative variables.

Non-parametric Kruskal Wallis and Mann Whitney tests were used to assess the correlation of OCT4, SOX2 and NANOG expression with the clinical and pathological parameters of patients. The association of expression of OCT4, SOX2, and NANOG with one another was analyzed using the Spearman's correlation test. The data were statistically analyzed using SPSS version 22 (SPSS Inc., IL, USA) and $\mathrm{P}<0.05$ was considered statistically significant.

\section{Results}

This study was conducted on 30 OSCC and 30 non-tumoral adjacent tissue specimens. The mean age of patients was $62.53 \pm 13.55$ years. Tongue was the most common primary site of tumor (46.7\%). Demographic and data regarding the staging of tumors and the pathological characteristics of OSCCs (pathological subtype, tumor grade, presence of necrosis and lymph node, vascular and perineural invasion) in patients were presented in Table 1 . The majority of patients were in stage IV (53.4\%) while there was no case with metastasis in patients at stage IV.

\section{Expression of CSC markers in tumoral and non-tumoral} tissues

Expression of OCT4 $(\mathrm{P}=0.505)$ and NANOG $(\mathrm{P}=0.681)$ was not significantly different between tumoral and non-tumoral tissues. The results showed that the expression of SOX2 gene in the adjacent non-tumoral tissue was significantly higher than that in the tumoral tissue. Expression of SOX2 in the tumoral tissue was 0.169 times its expression in the adjacent non-tumoral tissue $(\mathrm{P}=0.049$; Table 2$)$. The association between SOX2, OCT4 and NANOG expression in the tumoral and adjacent non-tumoral tissues was evaluated and it was found that expression of SOX2 was significantly correlated with the expression of NANOG (Spearman's correlation test, $\mathrm{P}=0.024, \mathrm{r}=0.411$ ).

Association of expression of CSC markers with clinical and pathological characteristics of patients

The expression of SOX2 was not significantly correlated with clinical or pathological characteristics of patients according to the Kruskal Wallis and Mann Whitney tests. Also, the expression of OCT4 and NANOG were not significantly correlated with clinical or pathological characteristics of patients (Table 3 ).

\section{Discussion}

While the CSC theory has been proven in leukemia (Griffin and Lowenberg, 1986) and breast cancer (Aktas et al., 2009; Fillmore and Kuperwasser, 2008), studies on the role of CSC in the development and progression of oral cancer is still in their infancy. Several researchers have focused on studying the expression of CSC markers and their prognostic value in normal oral mucosa and OSCC. OCT4, SOX2, and NANOG, the core transcription factors known for their regulatory role in the pluripotency of embryonic stem cell, have been largely implicated as the marker of CSC in different malignancies (Chiou et al., 2008; Vaiphei et al., 2014). However, the oncogenic role and the clinical significance of these CSC genes have not been well investigated in the head and neck SCCs as well as OSCC. In the present study, we evaluated the expression of CSC markers of OCT4, SOX2, and NANOG in OSCC versus the adjacent non-tumoral

Table 1. Demographic and Pathologic Information of OSCC Patients

\begin{tabular}{|c|c|c|}
\hline Variable & & No. of patients \\
\hline \multirow[t]{2}{*}{ Age } & $\leq 60$ years & 13 \\
\hline & $>60$ years & 17 \\
\hline \multirow[t]{2}{*}{ Gender } & Male & 14 \\
\hline & Female & 16 \\
\hline \multirow[t]{5}{*}{ Ethnicity } & Persian & 8 \\
\hline & Middle East & 14 \\
\hline & Azerbaijani & 3 \\
\hline & Arab & 2 \\
\hline & Unknown & 3 \\
\hline \multirow[t]{3}{*}{ Family History } & Present & 10 \\
\hline & Absent & 20 \\
\hline & Tongue & 14 \\
\hline \multirow[t]{5}{*}{ Primary sit of tumor } & Lips \& Buccal Mucosa & 7 \\
\hline & Gingivae & 2 \\
\hline & Mouth Floor & 3 \\
\hline & Parotid gland & 1 \\
\hline & Unknown & 3 \\
\hline \multirow[t]{3}{*}{ Tumor Size $(\mathrm{cm})$} & $\leq 3$ & 11 \\
\hline & $3.1-6$ & 14 \\
\hline & $>6$ & 5 \\
\hline \multirow[t]{4}{*}{ T Status } & $\mathrm{T} 1$ & 3 \\
\hline & $\mathrm{T} 2$ & 10 \\
\hline & $\mathrm{T} 3$ & 6 \\
\hline & $\mathrm{T} 4$ & 11 \\
\hline \multirow[t]{4}{*}{ N Status } & $\mathrm{Nx}$ & 10 \\
\hline & No & 12 \\
\hline & N1 & 3 \\
\hline & $\mathrm{N} 2$ & 5 \\
\hline \multirow[t]{2}{*}{ Metastasis } & M0 & 30 \\
\hline & M1 & 0 \\
\hline \multirow[t]{4}{*}{ TNM Stage } & I & 3 \\
\hline & II & 7 \\
\hline & II & 4 \\
\hline & IV & 16 \\
\hline \multirow[t]{2}{*}{ Pathologic Subtype } & Keratinized & 2 \\
\hline & Non-keratinized & 28 \\
\hline \multirow[t]{8}{*}{ Pathologic Grade } & I & 15 \\
\hline & II & 13 \\
\hline & III & 1 \\
\hline & Unknown & 1 \\
\hline & Necrosis & 2 \\
\hline & Lymphatic Invasion & 4 \\
\hline & Vascular Invasion & 6 \\
\hline & Perineural Invasion & 8 \\
\hline
\end{tabular}

Asian Pacific Journal of Cancer Prevention, Vol 201651 
Table 2. Comparison of the Expression of SOX2, OCT4 and NANOG in the Tumoral and Non-tumoral Tissues

\begin{tabular}{lcccc}
\hline Gene & Value & $\Delta$ CT of the tumoral tissue & $\Delta$ CT of the adjacent healthy tissue & P value* \\
\hline SOX2 & Mean \pm SD & $5.55 \pm 5.08$ & $2.99 \pm 4.80$ & 0.049 \\
& Median (interquartile range) & $4.5(1.14-9.09)$ & $2.5(1.17-7.0)$ & 0.505 \\
OCT4 & Mean \pm SD & $3.30 \pm 5.22$ & $2.45 \pm 4.55$ & 0.681 \\
& Median (interquartile range) & $3.77(0.05-7.23)$ & $3.54(0.13-5.73)$ & $4.91 \pm 5.40$ \\
NANOG & Mean \pm SD & $5.45 \pm 4.75$ & $5.79(2.71 \pm 7.99)$ & \\
& Median (interquartile range) & $4.25(1.86 \pm 9.39)$ &
\end{tabular}

*, T-test (REST software)

Table 3. Association of SOX2, OCT4 and NANOG with Clinical and Pathological Characteristics of Patients

\begin{tabular}{|c|c|c|c|c|c|c|c|}
\hline \multirow[b]{2}{*}{ Variable } & \multicolumn{3}{|c|}{ SOX2 expression } & \multirow{2}{*}{$\begin{array}{c}\text { OCT4 expression } \\
\text { Median }\end{array}$} & \multicolumn{3}{|c|}{ NANOG expression } \\
\hline & Classification & Median & $P$ value & & $P$ value & Median & $P$ value \\
\hline \multirow[t]{3}{*}{$\mathrm{N}$} & N0 & 16.0 & $0.636^{*}$ & 4.0 & $0.374 *$ & 7.81 & $0.572 *$ \\
\hline & N1 & 0.80 & & 0.01 & & 2.00 & \\
\hline & $\mathrm{N} 2$ & 0.03 & & 0.60 & & 0.50 & \\
\hline \multirow[t]{4}{*}{ TNM } & Stage I & 5.31 & $0.367 *$ & 11.79 & $0.658^{*}$ & 7.31 & $0.399 *$ \\
\hline & Stage II & 21.54 & & 3.47 & & 13.23 & \\
\hline & Stage III & 0.80 & & 0.01 & & 2.00 & \\
\hline & Stage IV & 0.01 & & 0.39 & & 0.37 & \\
\hline \multirow[t]{2}{*}{ Histological grade } & Grade I & 0.27 & $0.458^{* *}$ & 0.76 & $0.945^{* *}$ & 0.16 & $0.146^{* *}$ \\
\hline & Grade II /III & 0.24 & & 1.94 & & 3.97 & \\
\hline \multirow[t]{2}{*}{ Lymphatic invasion } & Yes & 0.53 & $0.803 * *$ & 0.01 & $0.567^{* *}$ & 2.0 & $0.471 * *$ \\
\hline & No & 0.01 & & 1.90 & & 4.02 & \\
\hline
\end{tabular}

*, Kruskal Wallis test; **, Mann Whitney test

tissue and showed significantly higher expression of SOX2 in the adjacent non-tumoral tissues compared to the tumoral tissue.

Carcinogenesis of oral cancer includes several stages, in which normal cells are progressively transformed to cancer cells. Stem and progenitor cells reside in tissue are the most common targets of genetic transformation and after going through several genetic mutations could convert to CSCs. Studies on the progressive development of different cancer have shown that in normal tissue adjacent to tumoral and preneoplastic tissues, the expression of CSC markers have increased that indicate the early molecular changes in normal tumor-adjacent cells and the possible role of CSCs in the process of carcinogenesis (Krishnamurthy and Nör, 2012). Therefore, measuring CSC markers could enhance the diagnosis accuracy of disease at the early stages and can be used as a prognostic indicator.

Sex-determining region Y-box 2 (SOX2) is a transcription factor involved in pluripotency and self-renewal of embryonic stem cells. In CSC, SOX-2 plays an essential role in the survival of malignant squamous cell and could protect CSCs against apoptosis (Hussenet and du Manoir, 2010).

Although our results showed increased expression of SOX 2 in adjacent non-tumoral tissue, there are controversies regarding the expression of SOX2 and its clinical usefulness between different studies on OSCC. Qiao et al., (2014) showed the simultaneous expression of OCT4 and SOX2 in transforming oral mucosal samples in rat models as well as pre-neoplastic and neoplastic human tissue samples. Although the expression of these two markers in metastatic samples was lower than that in the primary tumor sites, their results showed no expression in normal tissues. In agreement with our results, Fu et al, studied 436 tumoral samples, 362 adjacent non-tumoral samples and 76 normal tissue samples (uvula) and revealed that the expression of SOX2 was higher in the adjacent non-tumoral tissue compared to the tumoral tissues (Fu et al., 2016). The same results were reported in other malignancies. Li et al. demonstrated that expression of SOX2 in the gastric mucosa and intestinal metaplasia was higher than that in stomach cancer cells ( $\mathrm{Li}$ et al., 2004). Also, the high expression of SOX 2 was found in normal bronchial mucosa compared to squamous dysplasia of the lungs (Yuan et al., 2010).

It has been suggested that increased expression of SOX2 may be involved in primary transformation and carcinogenesis of squamous tumors in the process of hyperplasia and dysplasia. Thus, increased expression of SOX2 in the non-tumoral adjacent tissues may indicate primary molecular changes similar to those occurring in preneoplastic tissues. Alternately, such a higher expression in the adjacent non-tumoral tissue may simply indicate the presence of normal squamous precursor cells with high expression of SOX2, which are more abundant in the adjacent non-tumoral tissue compared to the tumoral tissue (Fu et al., 2016).

Our results also showed no significant difference in the expression of OCT4 and NANOG between the OSCC 
and adjacent non-tumoral tissue. In contrast to our results, Vaiphei et al. study on esophageal SCC, showed increased expression of OCT4 in the esophageal mucosa adjacent to an area of mild dysplasia and basal hyperplasia (Vaiphei et al., 2014). In their study, normal mucosal biopsies were negative for OCT4.

Study the expression of SOX2, NANOG and OCT4 in our study showed that there existed no association between CSC markers and lymph node metastasis, tumor stage, pathological grade and lymphatic invasion. The significance of SOX2 in lymph node metastasis and distant metastasis has been previously evaluated and shown that higher expression of SOX2 is correlated with lymph node metastasis, distant metastasis and poorer prognosis of colorectal cancer (Li et al., 2015). Also, evidence has shown that SOX2 knockdown induces mesenchymal-epithelial transition, which is the reverse process of epithelial-mesenchymal transition. In the latter process, cells become polarized and mobile; the characteristic that is necessary for invasion and metastasis in epithelial malignancies. It has been shown that SOX2-positive tumors, maintain such characteristic during lymph node metastasis. This finding may further indicate that SOX2-positive tumors have higher capability and likelihood of lymph node metastasis. The expression of SOX2 at the site of lymph node metastasis compared to primary tumor site has been investigated in other malignancies (Michifuri et al., 2012). Accordingly, decreased expression of SOX2 is significantly associated with decreased angiogenesis and lymphangiogenesis in breast cancer (Chen et al., 2008). Another study on lung cancer indicated the association of SOX2 with lymphatic metastasis (Ren et al., 2016).

Studies on head and neck SCC also demonstrated a significant association between SOX2 expression and lymph node metastasis (Michifuri et al., 2012; Ren et al., 2016). However, there are few studies reporting that there is no link between SOX2 expression and tumor stage, lymphatic metastasis or distant metastasis in this type of SCC. Similar to head and neck SCC, the role of SOX2 in OSCC has not yet been fully elucidated. It has been demonstrated that SOX2 in oral cancer has two cytoplasmic staining patterns known as disseminated and peripheral. The disseminated pattern was significantly associated with metastasis (Nichols et al., 1998).

While Neumann et al. showed that higher expression of SOX2 at the site of primary tumor correlate with poorer prognosis and lymph node metastasis (Neumann etal., 2011), others demonstrated a significant correlation between increased expression of SOX2 and the absence of lymph node metastasis (Züllig et al., 2013). They suggested that the expression of SOX2 is a suitable indicator of the absence of regional lymph nodes metastasis in oral cancer. Using immunohistochemistry, Fu et al. evaluated the expression of OCT4, SOX2, and NANOG in oral cancer and showed that the expression of OCT4 and SOX2 increased with decreased tumor stage and size as well as the absence of lymph node metastasis (Fu et al., 2016). In our study, expression of OCT4, SOX2 and NANOG genes was slightly, but not significantly, higher in patients without lymph node metastasis.
To the best of our knowledge, this study is the first one to measure CSC markers at RNA level and that use of relatively large sample size is the strength point of our study. One limitation of our study was the absence of healthy tissue control for evaluating the expression of CSC markers, therefore, including normal sample is suggested for future studies. Studies on association of prognosis and survival rate with the expression of CSC markers could be an interesting topic for further researches in this line.

In conclusion, within the limitations of this study, the results showed that expression of SOX2 was significantly higher in the adjacent non-tumoral tissue compared to the tumoral tissue. The expression of OCT4 and NANOG was not significantly different between the tumoral and non-tumoral adjacent tissues. These findings suggest the possible use of SOX2 for early detection of OSCC.

\section{Acknowledgements}

The authors would like to thank Dr Ahmad Reza Shamshiri for assistance with statistical analysis. The present research project was granted by Dental Research Center, Dentistry Research Institute, Tehran University of Medical Sciences.

\section{References}

Aktas B, Tewes M, Fehm T, et al (2009). Stem cell and epithelial-mesenchymal transition markers are frequently overexpressed in circulating tumor cells of metastatic breast cancer patients. Breast Cancer Res, 11, R46.

Al-Hajj M, Clarke MF (2004). Self-renewal and solid tumor stem cells. Oncogene, 23, 7274-82.

Allegra A, Alonci A, Penna G, et al (2014). The cancer stem cell hypothesis: a guide to potential molecular targets. Cancer Invest, 32, 470-95.

Bau DT, Tsai CW, Lin CC, Tsai RY, Tsai MH (2011). Association of alpha B-crystallin genotypes with oral cancer susceptibility, survival, and recurrence in Taiwan. PLoS One, 6, e16374

Bernier J (2011). Head and neck cancer: multimodality management. Springer Science and Business Media press, New York. ?

Chen Y, Shi L, Zhang L, et al (2008). The molecular mechanism governing the oncogenic potential of SOX2 in breast cancer. J Biol Chem, 283, 17969-78.

Chiou SH, Yu CC, Huang CY, et al (2008). Positive correlations of Oct-4 and Nanog in oral cancer stem-like cells and high-grade oral squamous cell carcinoma. Clin Cancer Res, 14, 4085-95.

Fillmore CM, Kuperwasser C (2008). Human breast cancer cell lines contain stem-like cells that self-renew, give rise to phenotypically diverse progeny and survive chemotherapy. Breast Cancer Res, 10, R25.

Fu TY, Hsieh IC, Cheng JT, et al (2016). Association of OCT4, SOX2, and NANOG expression with oral squamous cell carcinoma progression. J Oral Pathol Med, 45, 89-95.

Geum DH, Roh YC, Yoon SY, et al (2013). The impact factors on 5-year survival rate in patients operated with oral cancer. J Korean Assoc Oral Maxillofac Surg, 39, 207-16.

Griffin J, Lowenberg B (1986). Clonogenic cells in acute myeloblastic leukemia. Blood, 68, 1185-95.

Hussenet T, du Manoir S (2010). SOX2 in squamous cell 
carcinoma: amplifying a pleiotropic oncogene along carcinogenesis. Cell Cycle, 9, 1480-86.

Islam F, Qiao B, Smith RA, Gopalan V, Lam AK (2015). Cancer stem cell: fundamental experimental pathological concepts and updates. Exp Mol Pathol, 98, 184-91.

Krishnamurthy S, Nör J (2012). Head and neck cancer stem cells. J Dent Res, 91, 334-40

Li XL, Eishi Y, Bai YQ, et al (2004). Expression of the SRY-related HMG box protein SOX2 in human gastric carcinoma. Int J Oncol, 24, 257-63

Li Y, Lv Z, He G, et al (2015). The SOX17/miR-371-5p/SOX2 axis inhibits EMT, stem cell properties and metastasis in colorectal cancer. Oncotarget, 6, 9099-112.

Liu SA, Wong YK, Lin JC, et al (2007). Impact of recurrence interval on survival of oral cavity squamous cell carcinoma patients after local relapse. Otolaryngol Head Neck Surg, 136, 112-8.

Michifuri Y, Hirohashi Y, Torigoe T, et al (2012). High expression of ALDH1 and SOX2 diffuse staining pattern of oral squamous cell carcinomas correlates to lymph node metastasis. Pathol Int, 62, 684-9.

Neumann J, Bahr F, Horst D, et al (2011). SOX2 expression correlates with lymph-node metastases and distant spread in right-sided colon cancer. BMC Cancer, 11, 518.

Nichols J, Zevnik B, Anastassiadis K, et al (1998). Formation of pluripotent stem cells in the mammalian embryo depends on the POU transcription factor Oct4. Cell, 95, 379-91.

Qiao B, He B, Cai J, Yang W (2014). The expression profile of Oct 4 and Sox 2 in the carcinogenesis of oral mucosa. Int $J$ Clin Exp Pathol, 7, 28-37.

Ren ZH, Zhang CP, Ji T (2016). Expression of SOX2 in oral squamous cell carcinoma and the association with lymph node metastasis. Oncol Lett, 11, 1973-79.

Reya T, Morrison SJ, Clarke MF, Weissman IL (2001). Stem cells, cancer, and cancer stem cells. Nature, 414, 105-11.

Sharma P, Saxena S, Aggarwal P (2010). Trends in the epidemiology of oral squamous cell carcinoma in Western UP: an institutional study. Indian J Dent Res, 21, 316-9.

Vaiphei K, Sinha SK, Kochhar R (2014). Comparative analysis of Oct4 in different histological subtypes of esophageal squamous cell carcinomas in different clinical conditions. Asian Pac J Cancer Prev, 15, 3519-24

Woolgar JA, Scott J, Vaughan ED, et al (1995). Survival, metastasis and recurrence of oral cancer in relation to pathological features. Ann R Coll Surg Engl, 77, 325-31.

Yu CC, Hu FW, Yu CH, Chou MY (2016). Targeting CD133 in the enhancement of chemosensitivity in oral squamous cell carcinoma-derived side population cancer stem cells. Head Neck, 38, e231-8.

Yuan P, Kadara H, Behrens C, et al (2010). Sex determining region Y-Box 2 (SOX2) is a potential cell-lineage gene highly expressed in the pathogenesis of squamous cell carcinomas of the lung. PloS One, 5, e9112.

Züllig L, Roessle M, Weber C, et al (2013). High sex determining region Y-box 2 expression is a negative predictor of occult lymph node metastasis in early squamous cell carcinomas of the oral cavity. Eur J Cancer, 49, 1915-22.

\section{(c) (1) ()}

This work is licensed under a Creative Commons AttributionNon Commercial 4.0 International License. 